\title{
Contribución al conocimiento de los Coccinellini micófagos (Coleoptera: Coccinellidae) en el departamento del Cusco, Perú
}

\section{Contribution to the knowledge of the mycophagous Coccinellini (Coleoptera: Coccinellidae) from the Cusco Department, Peru}

Abdhiel Bustamante-Navarrete *

https://orcid.org/0000-0001-8120-1274

abdhiel77@gmail.com

\section{*Corresponding author}

Universidad Nacional San Antonio Abad del Cusco, Colección Entomológica. Gabinete C-338, Pabellón C, Ciudad Universitaria de Perayoc. Av. de la Cultura, 733, Cusco, Perú.

\section{Citación}

Bustamante-Navarrete A. 2021. Contribución al conocimiento de los Coccinellini micófagos (Coleoptera: Coccinellidae) en el departamento del Cusco, Perú. Revista peruana de biología 28(2): e18852 (Mayo 2021). doi: http://dx.doi.org/10.15381/rpb.v28i2.18852

$\begin{array}{ll}\text { Presentado: } & 28 / 09 / 2020 \\ \text { Aceptado: } & 27 / 02 / 2021 \\ \text { Publicado online: } & 23 / 04 / 2021 \\ & \\ \text { Editor: } & \text { Diana Sllva }\end{array}$

\section{Resumen}

Se presenta una revisión de los ejemplares de Coccinellini micofagos procedentes de varias provincias del departamento del Cusco, en el sur oriente de Perú. Este grupo presenta actualmente nueve especies en el país, dentro de los géneros Oxytella Weise 1902, y Psyllobora Chevrolat 1836. En el material examinado se reporta la ocurrencia de cinco de ellas: Oxytella longula Weise, Psyllobora marshalli Crotch, P. abancayana Almeida, P. constantini González, Perla \& Almeida, y P. hybrida (Mulsant); además de otras tres especies, aún no identificadas, dentro del género Psyllobora. Para cada especie se presenta una diagnosis, fotografías y microfotografías de habito y estructuras genitales, e información sobre su distribución en Cusco.

\section{Abstract}

In this work, we presented a review of the specimens of mycophagous Coccinellini from various provinces of the Cusco Department, south east of Peru. This group currently has nine species in the country, within the genera Oxytella Weise 1902, and Psyllobora Chevrolat 1836. In the material examined the occurrence of five of them is reported: Oxytella longula Weise, Psyllobora marshalli Crotch, $P$. abancayana Almeida, P. constantini González, Perla \& Almeida, and P. hybrida (Mulsant); in addition to three other species, not yet identified, within the genus Psyllobora. For each species a diagnosis, photographs and photomicrographs of habit and genital structures, and information on their distribution in Cusco are presented.

Palabras clave:

Halyziini; micofagia; Psyllobora; Oxytella; insectos benéficos; organismos benéficos; control biológico; mariquitas; catarinas; coleópteros; Coccinellidae.

Keywords:

Halyziini; mycophagia; Psyllobora; Oxytella; beneficial insects; beneficial organisms; biological control; ladybirds; ladybugs; Coleoptera; Coccinellidae.

\section{Introducción}

Los géneros micófagos de Coccinellidae son un grupo cosmopolita que incluye especies que también pueden consumir tejido vegetal y ácaros (Sutherland \& Parella 2009). Análisis moleculares sugieren que este grupo surgió dentro de la tribu Coccinellini, mayormente afidófaga, y que a pesar de su morfología mandibular, relacionada con el hábito micófago, el grupo tiene características distintivas que apuntan más a que la micofagia es una condición derivada (Giorgi et al. 2009).

Mulsant (1850) estudió la tribu parafilética “Trimeres”, creada por Dejean (1837), y propuso la rama Halyziares que incluía los géneros Psyllobora Dejean, Halyzia Mulsant, y Thea Mulsant, como un primer intento de segregar taxonómicamente la micofagia en la familia. 
Chapuis (1876) consideró a Psyllobora como subgénero de Halyzia en el grupo Coccinellites, incluido a su vez dentro de Coccinélidos Afidófagos. Casey (1899) revisó la taxonomía de los coccinélidos norteamericanos y los organizó en 16 tribus, una de las cuales era Psylloborini, y sucesivos autores (Korschefsky 1932, Sasaji 1968, Kovar 1996, Kuznetsov 1997) mantuvieron el nombre tribal, considerando a Halyziini como sinónimo junior (Kitano 2019).

El taxón Halyziini (de Halyziares Mulsant) fue resucitado por Pakaluk et al. (1994) y adoptado en la clasificación de la familia de Vandenberg (2002), junto con Coccinellini, bajo Coccinellinae. Posteriormente Bouchard et al. (2011) sinonimizaron Halyziini con la tribu Coccinellini. En el Perú, los coccinellini micofagos están representados por los géneros Oxytella Weise 1902, y Psyllobora Chevrolat 1836 (González 2015).

El género Oxytella Weise fue descrito para albergar a las especies Oxytella subcordata, de Brasil, y O. longula, de Perú, y así es citado por Blackwelder (1945). Fue estudiado, dentro de Psylloborini, por Almeida (1992), y luego incluido dentro de Halyziini por Kuztnezov (1997). Este género se caracteriza por tener mandíbulas multidentadas, caracter asociado al hábito alimenticio micófago y compartido con Psyllobora Chevrolat y Neohalyzia Crotch, cuyas diferencias con Oxytella son superficiales y ameritan una revisión (González et al. 2017). El género está presente en Brasil (Blackwelder 1945; Weise 1902), y en Perú, donde está representado por la especie endémica O. longula (González 2015).

El género Psyllobora Chevrolat 1836, al igual que Oxytella, presenta mandíbulas multidentadas asociadas a una dieta micófaga (González et al. 2017). Este género es tradicionalmente considerado cosmopolita; sin embargo, Almeida (1992) consideró a las especies del viejo mundo dentro de Thea Mulsant y restringió Psyllobora al continente americano. Sucesivos trabajos de Almeida (1985, 1991a, 1991b, 1991c, 1992), Almeida \& Marinoni (1983) y González et al. (2017) incrementaron el número de especies del género, que actualmente comprende unas 60 especies distribuidas en Brasil, Guayana Francesa, Bolivia, México, Colombia, Venezuela, Guatemala, Paraguay, Surinam, Jamaica, Puerto Rico, Cuba, Argentina, Perú, Chile, Honduras, Nicaragua, Ecuador, Panamá, y en el Perú, donde existen ocho especies (Blackwelder 1945, González 2007, González et al. 2017). Ante la ausencia de estudios sobre aparatos genitales para la mayoría de las especies, "la identificación queda librada al análisis de las manchas elitrales, carácter no siempre estable ni preciso" (González et al. 2017).

El objetivo del presente estudio es dar a conocer la diversidad de los Coccinellini micófagos en el departamento del Cusco, como línea de base para estudios futuros acerca de su potencial como controladores biológicos.

\section{Material y métodos}

Se examinaron especímenes de los géneros Psyllobora Chevrolat y Oxytella Weise, depositados en la Co- lección Entomológica de la Universidad Nacional San Antonio Abad del Cusco, Perú (CEUC-UNSAAC). Cada espécimen montado se encuentra junto a una o más etiquetas que contienen los datos de colecta. Para la identificación fue necesaria la extracción y procesamiento del aparato genital del macho, y en algunos casos el de la hembra, siguiendo el método de González (2018). Las genitalias extraídas fueron conservadas, dentro de microviales, con glicerina en los alfileres junto a su respectivo ejemplar.

El habitus de cada espécimen fue fotografiado con una cámara AxioCam $®$ ICc5 montada en un microscopio estereoscopio Discovery® V20; las estructuras genitales fueron fotografiadas con una cámara Truechrome® II instalada en un microscopio Novel ${ }^{\circledR} \mathrm{N}-800 \mathrm{~m}$. En ambos casos, las fotografías fueron hechas en enfoque secuencial y combinadas usando el programa Helicon Focus ${ }^{\circledR}$.

Para cada especie se presenta una diagnosis, distribución, fotografías de su habitus, y microfotografías del aparato genital. Para la información del material examinado se transcribieron, literalmente, los datos de las etiquetas, la información faltante y/o relevante fue añadida entre corchetes [...]. Todos los especímenes estudiados se encuentran depositados en la Colección Entomológica de la Universidad Nacional San Antonio Abad del Cusco, Perú. Un mapa con las localidades de colecta de las especies de Coccinellini reportadas en este trabajo se presenta en la Figura 9.

\section{Resultados \\ Lista comentada}

\section{Oxytella longula Weise 1902}

(Figs. 1a, 1b, 1c)

Longitud: $4.5 \mathrm{~mm}$. Forma oval, con los élitros en forma de ojiva, de color amarillento oscuro con manchas negras. Pronoto con cuatro manchas en semicírculo, y una mancha en la base, entre las anteriores. Élitros con siete manchas negras; dos cercanas a la base, una humeral pequeña y la otra entre ésta y el escutelo; tres en línea transversal irregular a la mitad del largo, la más grande sutural, la menor cerca del borde extendiéndose sobre el reborde elitral; las dos últimas a los 3/4 del largo, la más grande cercana a la sutura (Fig. 1a). Borde sutural negro.

Distribución: PERÚ (Cusco) (Almeida, 1992). Localidad tipo: Perú, Marcapata, Cusco (Almeida, 1992).

Material examinado: ( 1 ej.) Esperanza/

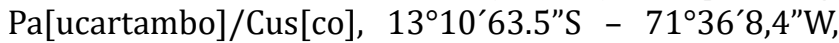
Juan F. Costa [col], 2821 m, (Det. G. González 2012).

\section{Psyllobora marshalli Crotch 1874}

(Figs. 2a, 2b, 2c)

Longitud: $4 \mathrm{~mm}$. Forma oval y de color amarillo pajizo con manchas negras. Pronoto con una gran mancha basal negra la cual deja un borde lateral y delantero 
amarillos. Élitros con manchas negras fundidas las que cubren todo el disco y tres manchas laterales al tercio, dos tercios y cerca del ápice, de las cuales solo la primera toca el borde. Además, hay un punto amarillo en el medio del disco a 1/4 del largo y una manchita común sutural amarilla a nivel de los 2/3 del largo (Fig. 2a).

Distribución: GUAYANA FRANCESA, PERÚ (Blackwelder 1945, González 2007). Localidad tipo: Guayana Francesa (Crotch, 1874).

Material examinado: (1 ej.) Trocha Unión, 2750m, Kosñipata/Pa[ucartambo]/Cus[co], 31-12-[20]01, Juan F. Costa [col], (Det. G. González 2010).

\section{Psyllobora abancayana Almeida 1991}

(Figs. 3a, 3b, 3c)

Longitud: 4.8 - $5.2 \mathrm{~mm}$. Forma oval bastante ancha, poco convexa. Cabeza marrón, pronoto marrón amarillento con cuatro pequeñas manchitas negras en semicírculo alrededor del medio de la base. Escutelo negro. Élitros con ocho manchas negras, pequeñas casi punctiformes (1:5:1:1), a 1/8,1/2,3/4, y 7/8 del largo, las 5 centrales formando una línea transversal que no toca la sutura, pero si el borde lateral (Fig. 3a). Mancha negra en la parte media de la epipleura.

Distribución: PERÚ (Apurímac, Ancash, Cusco) (Almeida 1991b; González 2007). Localidad tipo: Abancay, Perú (Almeida, 1991b).

Especie descrita con material procedente de Abancay (Apurímac).

Material examinado: (1 ej.): Limatambo 2500, Limatambo/Anta/Cus[co], 12.08.2003, A. Bustamante [col]; (2 ejs.): PERÚ: Mollepata, 3121m, Anta, Cusco, 17/03/2017, -13.4883 / -72.7476, A. Elme \& E. Marquina [col]; (2 ej.) PERÚ: Cusco/Quillabamba, S[an]ta. Teresa - Choquequirao, -13.3928 / -72.8642, 5-20/xi/2017, 2700 m, A. Laurel [col]; (2 ej.) PERÚ, Cusco, La Convención, Santa Teresa, Choquequirao, -13.3928 -72.8642, diciembre 2017, 2700m, A. Laurel [col].

\section{Psyllobora constantini González et al. 2017}

(Figs. 4a, 4b, 4c)

Longitud: $5 \mathrm{~mm}$. Forma ovalar, ojival hacia atrás y de color marfil con manchas negras. Cabeza negra con mancha marfil en la frente. Pronoto con una mancha negra en la base que se extiende hasta los 3/4 del largo, sin llegar al ápice y que deja todo el borde lateral, delantero y una línea longitudinal que corta por el medio la mancha negra, de color marfil. Escutelo negro. Élitros con ocho manchas cada uno, distribuidas en cuatro filas (2:3:2:1), todas aisladas de los bordes y entre sí, excepto la tercera mancha de la segunda fila, que toca el borde lateral. Reborde elitral y base del reborde pronotal negros (Fig. 4a).

Distribución: PERÚ (Huánuco) (González et al. 2017). Localidad tipo: Huánuco, Perú (González et al. 2017).
Se caracteriza por su tamaño, mayor al de la mayoría de las especies del género, y por su diseño particular, con ocho manchas en disposición única (González et al. 2017). Primer registro de la especie para el departamento del Cusco, y que amplia su distribución al sur de la localidad tipo.

Material examinado: (1 ej.) "Quillabamba”, LC [La Convención]/Cusco, diciembre 1971, J. Escalante [col].

\section{Psyllobora hybrida (Mulsant 1850)}

(Figs. 5a, 5b, 5c)

Longitud: $4.5 \mathrm{~mm}$. Forma oval y de color amarillo marfil con manchas castañas, los bordes transparentes. Pronoto con cuatro manchas en total, dos basales y dos discales. Élitros con ocho manchas cada uno, cuatro en la mitad anterior y cuatro en la mitad posterior, todas en distribución irregular, sin tocar los bordes laterales ni la sutura. En la mitad anterior, una gran mancha sutural, alargada y abarcando hasta casi la mitad del ancho elitral, y con una línea clara en el medio de la mancha, una mancha pequeña humeral cerca al borde elitral, y dos manchas subtriangulares entre la mancha grande y el borde elitral, detrás de la anterior. En la mitad posterior, una mancha en el borde lateral y otra en el borde sutural, y entre ambas dos manchas subtriangulares una detrás de la otra (Fig. 5a).

Distribución: ARGENTINA, BRASIL, PARAGUAY (Almeida 1985, González 2007). Localidad tipo: Matto Grosso, Brasil (Almeida, 1985).

Almeida (1985) describió la genitalia del macho de esta especie.

Material examinado: (1 ej.): Isilluyoc, Sambaray Alto, Santa Ana, La Convención, [Cusco], 1450 msnm, T-1 [transecto], 28.04.[20]14, [sobre] cítricos, Fredy J. Hurtado M. [col.].

\section{Psyllobora sp. 1}

(Figs. 6a, 6b, 6c, 6d)

Longitud: $2.7-3 \mathrm{~mm}$. Color marfil con manchas marrones. Pronoto con una manchita basal y cuatro en semicírculo. Élitros con múltiples manchas fusionadas, siendo visibles las dos primeras, cerca de la base elitral (2:3:3:1:1). La tercera tocando el borde lateral, la cuarta y la séptima agrandadas y fusionadas. La octava se extiende hacia la décima tocando el borde sutural. El color de fondo del marfil al amarillo marrón. Manchas marrones a oscuras casi negras. Todas las manchas pueden estar completamente fusionadas (Fig. 6a).

Material examinado: (4 ej.) Huayllapampa, 16.02. [20]08, S[a]n. Jerónimo/Cus[co], 13³2'01”S 7152’00”W, M. Puente de la Vega [col]; (1 ej.) Huayllapampa, 16.02 . [20]08, S[a]n. Jerónimo/Cus[co], 13³2'01”S 7152’00”W, A. Contreras [col]; (1 ej.) Perayoc - Cusco, -13.31 -71.57, 3365 m, 22/08/[20]13, Mondragón - Montoya [col]; (1 ej.) PERÚ: CU[SCO]. Kayra (INV) [Invernadero?], 1384’00.00" S / 7164’00.00" W, 3320m, 28.XI.2013, E. Mondragón., T. 
Montoya [col]; (1 ej.) PERÚ: CU[SCO]. K'ayra (INV) [Invernadero?], $13^{\circ} 84^{\prime} 00.00^{\prime \prime} \mathrm{S} / 7^{\circ} 64^{\prime} 00.00^{\prime \prime} \mathrm{W}, 28 . X I .2013$, E. Mondragón., T. Montoya [col].

\section{Psyllobora sp. 2}

(Figs. 7a, 7b, 7c)

Longitud: $3.5-4.2 \mathrm{~mm}$. Pronoto con cuatro manchas en semicírculo. Élitros con nueve manchas oscuras (2:3:3:1). La mancha 3 , subtriangular, puede fusionarse con el borde sutural. El color de fondo varía desde el marfil hasta el amarillo marrón. Las manchas pueden variar de un marrón claro a oscuro. Las manchas pronotales delanteras a veces unidas en una gran mancha triangular (Fig. 7a).

Los ejemplares están etiquetados como "Psyllobora lutescens Crotch"

Material examinado: (1 ej.): Isilluyoc, Sambaray Alto, Santa Ana, La Convención, 1450 msnm, T-1 [transecto], 28.04.[20]14, cítricos, Fredy J. Hurtado M. [col], "Psyllobora lutescens Crotch"; (1 ej.) Perú, Potrero, Santa Ana, La Convención, Julio 2017, [sobre] limón, A. Bustamante [col], "Psyllobora lutescens Crotch".

\section{Psyllobora sp. 3}

(Figs. 8a, 8b, 8c, 8d)

Longitud: $2.5-3 \mathrm{~mm}$. Forma ovalada y poco convexa. Cabeza y pronoto marrón amarillento, el último con cinco manchas marrón oscuro, cuatro en semicírculo alrededor de la quinta, en el centro de la base. Escutelo marrón oscuro. Élitros marrón amarillento, con 10 manchas marrones $(2: 3: 1: 2: 2)$, distancia entre las manchas de $1 / 4$ a $1 / 2$ veces el tamaño de las manchas. Mancha cuatro subtriangular, la seis-siete-ocho en semicírculo alrededor de la novena, y la novena alargada y oblicua hacia la sutura (Fig. 8a).

Los ejemplares están etiquetados como "Psyllobora luctuosa Mulsant"

Material examinado: (2 ej.) $\mathrm{Km} 86$, Ollantaytambo, Urubamba, febrero 2010, A. Bustamante [col], "Psyllobora luctuosa Mulsant"; (1 ej.) Sangobatea, Echarate/LC[La Convención]/Cusco, 03.03.1972, J. Escalante [col], "Psyllobora luctuosa Mulsant"; (1 ej.) Macamango, 1050, Santa Ana/LC [La Convención]/Cusco, 10.09.1973, J. Escalante [col], "Psyllobora luctuosa Mulsant"; (1 ej.) PERÚ, Cusco, La Convención, Santa Teresa, Choquequirao, -13.3928 -72.8642, diciembre 2017, 2700 m, A. Laurel [col], "Psyllobora luctuosa Mulsant".

\section{Discusión}

En los últimos veinte años, múltiples estudios en América, Asia, y Europa han tratado sobre la biología de los coccinellidos micofagos y su potencial para ser utilizados como agentes de control biológico, especialmente del 'mildiu pulverulento' (Sutherland \& Parella 2009). El mildiú pulverulento es causado por hongos parásitos biotróficos obligados de una amplia gama de plantas hospedadoras, incluida la mayoría de las especies cultivadas, y su control requiere aplicaciones repetidas de fungicidas (Pérez-García et al. 2009), debido a su capacidad para desarrollar resistencia (McGrath 2001, Risco et al. 2018). El mildiu en el Perú está reportado en quinua, albahaca, y otros importantes cultivos (Leon et al. 2018; Risco et al. 2018).

En el Perú, especies de Psyllobora se han encontrado en banano en presencia de Tetranychus sp., Aleurodicus sp., y pseudococcidos (Miro-Agurto \& Castillo-Carrillo, 2010), y alimentándose de esporas de Oidium (Korytkowski \& Ojeda, 1970; García, 1983), al igual que lo reportado en Cuba, donde se observó la acción micófaga de Psyllobora nana Mulsant sobre Oidium bixae en varios cultivos, y se relacionó directamente la abundancia del coccinellido con la intensidad de infestación por mildiu (Veitía et al. 2007).

Los coccinélidos micófagos son una parte importante de los sistemas agrícolas en todo el mundo y la biología de la tribu se debe explorar más a fondo antes de que se pueda comprender completamente su verdadero potencial para el control biológico, en búsqueda de estrategias de gestión alternativas para el mildiu pulverulento.

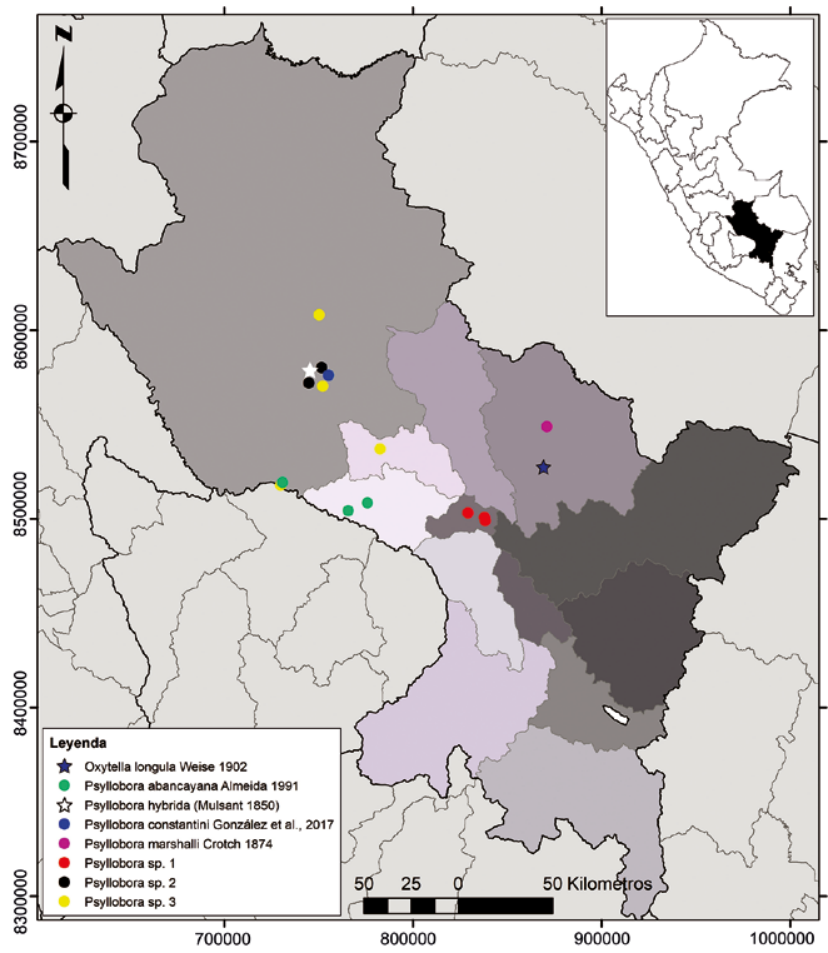

Figura 9. Localidades de colecta de las especies de Coccinellini micofagos en el departamento del Cusco. 

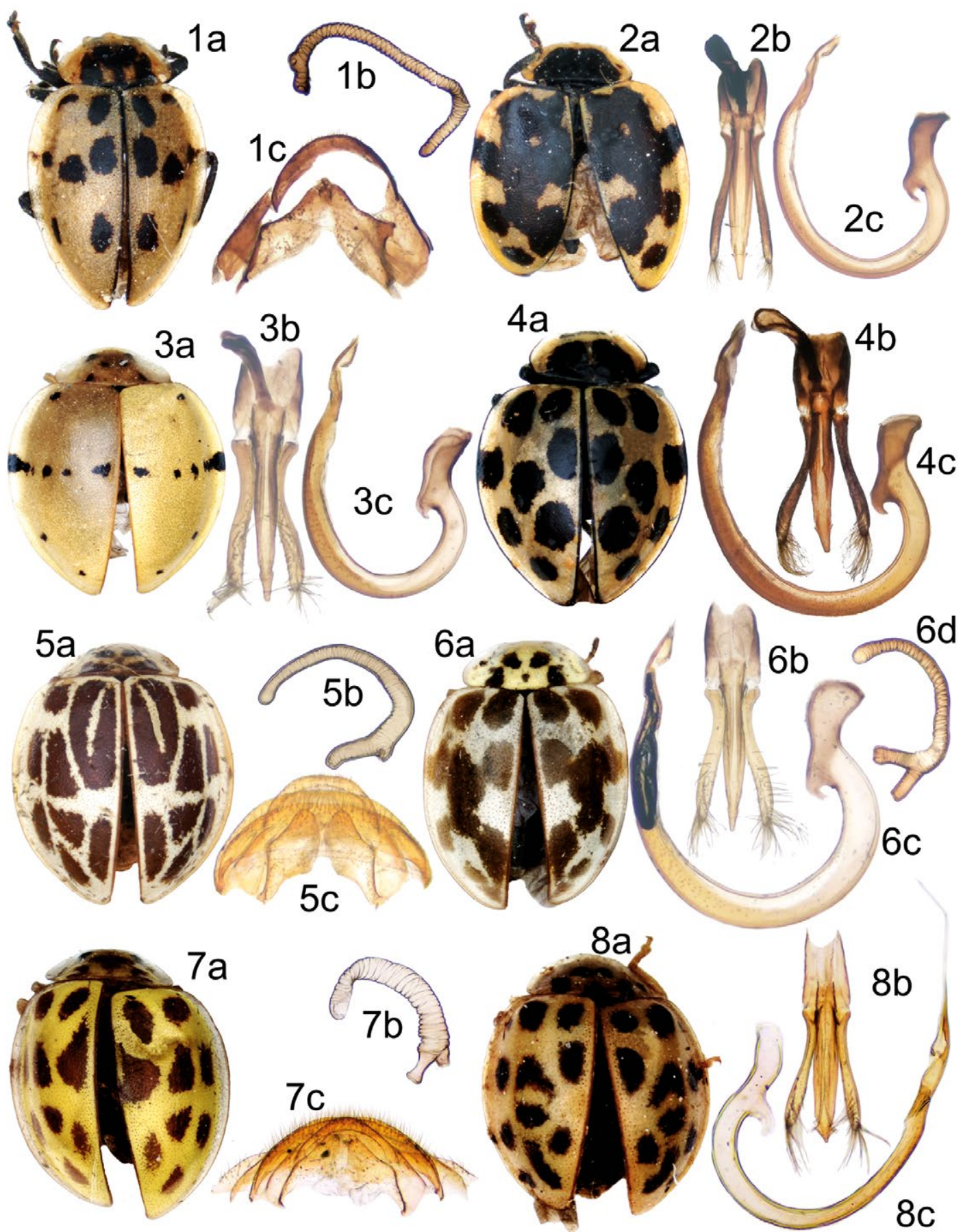

Figura 1. Oxytella longula Weise: a. habitus, b. espermateca, c. placa genital. Figura 2. Psyllobora marshalli Crotch: a. habitus, b. tegmen, c. penis. Figura 3. Psyllobora abancayana Almeida: a. habitus, b. tegmen, c. penis. Figura 4. Psyllobora constantini González et al.: a. habitus, b. tegmen, c. penis. Figura 5. Psyllobora hybrida (Mulsant): a. habitus, b. espermateca, c. placa genital. Figura 6. Psyllobora sp. 1: a. habitus, b. tegmen, c. penis, d. espermateca. Figura 7. Psyllobora sp. 2: a. habitus, b. espermateca, c. placa genital. Figura 8. Psyllobora sp. 3: a. habitus, b. tegmen, c. penis.

\section{Literatura citada}

Almeida L. 1985. Estudo de 17 espécies do genero Psyllobora Chevrolat, 1837 (Coleoptera - Coccinellidae). Acta Biológica Paranaense, 14, 47-102. https://doi. org/10.5380/abpr.v14i0.849

Almeida L. 1991a. Descrição de cinco espécies novas de Psyllobora Chevrolat, 1837 (Coleoptera, Coccinellidae, Psylloborini). Revista Brasileira de Zoologia, 7(3), 409-414. https://doi.org/10.1590/S010181751990000300018
Almeida L. 1991b. Duas espécies novas de psyllobora Chevrolat, 1837 (Coleoptera, Coccinellidae). Revista Brasileira de Zoologia, 7(3), 421-423. https://doi.org/10.1590/ S0101-81751990000300020

Almeida L. 1991c. Quatro novas espécies de Psyllobora Chevrolat, 1837 (Coleoptera, Coccinellidae). Revista Brasileira de Zoologia, 7(3), 415-420. https://doi. org/10.1590/S0101-81751990000300019

Almeida L. 1992. Notas e descrições em Psylloborini (Coleoptera, Coccinellidae). Revista Brasileira de Entomologia, 36(4), 759-765. 
Almeida L, Marinoni R. 1983. Contribuição ao conhecimento do gênero Psyllobora Chevrolat, 1837 (Coleoptera, Coccinellidae). Revista Brasileira de Entomología, 27(2), 165-175.

Blackwelder R. 1945. Checklist of the coleopterous insects of Mexico, Central America, The West Indies, and South America. Part 4. In Smithsonian Institution United States National Museum (Vol. 185, Issue 3). https://doi. org/10.5479/si.03629236.185.3

Bouchard P, Bousquet Y, Davies AE, Alonso-Zarazaga MA, Lawrence JF, Lyal CH, Newton AF, Reid CA, Schmitt M, Slipiński SA, Smith AB. 2011. Family-group names in Coleoptera (Insecta). ZooKeys, (88), 1-972. https:// doi.org/10.3897/zookeys.88.807

Casey TL. 1899. A revision of the American Coccinellidae. Journal of the New York Entomological Society 7, 71-169.

Chapuis F. 1876. Genera des Coleopteres ou expose methodique et critique de tous les genres proposes jusqu'ici dans cet ordre d'insectes. (Coccinellides). Paris 12, 149-259.

Crotch G. 1874. A revision of the coleopterous family Coccinellidae. E.W. Janson. https://doi.org/10.5962/bhl. title.8975

Dejean PFMA. 1837. Catalogue des coleopteres de la collection M. le Conte Dejean. Third ed. Paris, Mequignon-Marvis, pp. 1-458. https://doi.org/10.5962/t.173109

Garcia U. 1983. Ocurrencia, abundancia relativa e importancia de insectos y otros artropodos en el Crisantemo. Revista peruana de Entomología, 26(1): 31-39.

Giorgi JA, Vandenberg NJ, McHugh JV, Forrester JA, Ślipiński SA, Miller KB, Shapiro LR, Whiting M. 2009. The evolution of food preferences in Coccinellidae. Biological Control 51, 215-231. https://doi.org/10.1016/j.biocontrol.2009.05.019

González G. 2007. Los Coccinellidae de Perú. Disponible: https://www.coccinellidae.cl/paginasWebPeru/Paginas/InicioPeru.php. Acceso: 16 de setiembre de 2020.

González G. 2015. Beetles (Coleoptera) of Peru: A Survey of the Families. Coccinellidae. Journal of the Kansas Entomological Society, 88(2), 229-236. https://doi. org/10.2317/kent-88-02-229-236.1

González G. 2018. Aporte al conocimiento de la tribu Coccinellini (Coleoptera: Coccinellidae) en América del Sur. Revista Chilena de Entomología, 44(2), 169-206.

González G, Perla D, Almeida L. 2017. Descripción de tres notables nuevas especies del género Psyllobora Chevrolat (Coleoptera: Coccinellidae: Coccinellini) de Perú. Revista Chilena de Entomología 42, 81-90.

Kitano T. 2019.Taxonomical notes and descriptions of Japanese Halyziini (Coleoptera: Coccinellidae). Taxonomical series, 15(2):371-382.

Korytkowski C, Ojeda D. 1970. Catalogo del Museo de Entomología Univ. Nac. Pedro Ruiz Gallo, Lambayeque. 1(3) 84 pp.

Korschefsky R. 1932. Coleopterorum Catalogus. XVI \{120\}, Coccinellidae II. W. Junk. Berlin, pp. 565-570.

Kovar I. 1996. Phylogeny. En: Hodek, I., Honěk, A. (Eds.), Ecology of Coccinellidae. Kluwer Academic Publishers, Netherlands, pp. 19-31. 464 pp. https://doi. org/10.1007/978-94-017-1349-8_2

Kuznetsov VN. 1997. Lady Beetles of the Russian Far East. Memoir No.1. Center for Systematic Entomology, Sandhill Crane Press, Gainesville, $248 \mathrm{pp}$
Leon B, Ortiz N, Condori N, Chura E. 2018. Cepas de Trichoderma con capacidad endofitica sobre el control del mildiu (Peronospora variabilis Gäum.) y mejora del rendimiento de quinua. Revista de Investifaciones $\mathrm{Al}$ toandinas, 20(1):19-29. https://doi.org/10.18271/ ria.2018.327

McGrath MT. 2001. Fungicide resistance in cucurbit powdery mildew: experiences and challenges. Plant Disease, 85: 236-245. https://doi.org/10.1094/ PDIS.2001.85.3.236

Miro-Agurto J, Castillo-Carrillo P. 2010. Especies de "mariquitas" (Coleoptera:Coccinellidae) en los frutales de Tumbes. Revista peruana de Entomología, 46(1):21-29.

Mulsant ME. 1850. Species de Coleopteres trimeres securipalpes. Annales des Sciences physiques et naturelles de Lyon 2, 1104 pp. https://doi.org/10.5962/bhl.title.8953

Pakaluk J, Ślipiński SA, Lawrence JF. 1994. Current classification and familygroup names in Cucujoidea (Coleoptera). Genus 5, 223-268.

Pérez-Garcia A, Romero D, Fernández Ortuño D, López-Ruiz F De Vicente A, Torés JA. 2009. The powdery mildew fungus Podosphaera fusca (synonym Podosphaera xanthii), a constant threat to cucurbits. Molecular Plant Pathology, 10: 153-60. https://doi.org/10.1111/ j.1364-3703.2008.00527.x

Risco A, Apaza WE, Mattos LL. 2018. Identificacion del agente causal del mildiu de la albahaca y metodos para la estimación de su severidad. Anales Científicos 79(1):159-167.

Sasaji H. 1968. Phylogeny of the family Coccinellidae (Coleoptera). Etizenia. Occasional Publication of the Biological Laboratory Fukui University 35, 1-37.

Sutherland A, Parrella M. 2009. Mycophagy in Coccinellidae: Review and synthesis. Biological Control, 51, 284-293. https://doi.org/10.1016/j.biocontrol.2009.05.012

Vandenberg NJ. 2002. Family 93. Coccinellidae Latreille 1807. En: Arnett RHJr, Thomas MC, Skelley PE, Frank JH (Eds.). American Beetles. Polyphaga: Scarabaeoidea through Curculionoidea, vol. 2. CRC Press LLC, Boca Raton, FL, pp. 371-389.

Veitía M, Lopez D, Porras A, Montalvo JM. 2007. Estudio preliminar de la asociación Psyllobora nana Mulsant y mildiu en cultivos de plantas medicinales, condimenticas y otras en Cuba. Fotosanidad, 11(4):37-40.

Weise J. 1902. Coccinelliden aus Südamerika. III. Deutsche Entomologische Zeitschrift, 161-176.

Agradecimientos / Acknowledgments:

Al Dr. Erick Yábar Landa, responsable del Laboratorio de Entomología de la Escuela Profesional de Biología, Facultad de Ciencias de la Universidad Nacional San Antonio Abad del Cusco, por el uso de los equipos de microscopia y microfotografía, así como por el permiso para revisar la Colección Entomológica a su cargo.

Conflicto de intereses / Competing interests:

El autor declara que no incurre en conflictos de intereses.

Fuentes de financiamiento / Funding:

El autor declara que este trabajo no recibió financiación específica.

\section{Aspectos éticos / legales; Ethics / legals:}

Los autores declaran no haber incurrido en aspectos antiéticos. El presente estudio se efectuó con especímenes de colectas previas, y depositados en la Colección Entomológica de la Facultad de Ciencias de la Universidad Nacional San Antonio Abad del Cusco. 